\title{
NLRP3 Inflammasome Expression in Gingival Crevicular Fluid of Patients with Periodontitis and Chronic Hepatitis C
}

\author{
Petra Surlin $\mathbb{D}^{1},{ }^{1}$ Luminita Lazar $\mathbb{D}^{2},{ }^{2}$ Cerasella Sincar $\mathbb{D}^{3},{ }^{3}$ Dorin Nicolae Gheorghe $\mathbb{D}$, ${ }^{1}$ \\ Dora Maria Popescu $\left(\mathbb{D},{ }^{1}\right.$ Virgil Mihail Boldeanu ${ }^{(D)},{ }^{4}$ Allma Pitru $\mathbb{D}^{5},{ }^{5}$ Cristina Florescu $\mathbb{D}^{6}{ }^{6}$ \\ and Ion Rogoveanu ${ }^{7}{ }^{7}$ \\ ${ }^{1}$ Department of Periodontology, Faculty of Dental Medicine, University of Medicine and Pharmacy of Craiova, Romania \\ ${ }^{2}$ Department of Periodontology, Faculty of Dental Medicine, "George Emil Palade" University of Medicine and Pharmacy, \\ Targu Mures, Romania \\ ${ }^{3}$ Department of Periodontology, Faculty of Medicine, "Dunarea de Jos" University, Galati, Romania \\ ${ }^{4}$ Department of Immunology, Faculty of Medicine, University of Medicine and Pharmacy of Craiova, Romania \\ ${ }^{5}$ Department of Oral Pathology, Faculty of Dental Medicine, University of Medicine and Pharmacy of Craiova, Romania \\ ${ }^{6}$ Department of Internal Medicine and Cardiology, Faculty of Medicine, University of Medicine and Pharmacy of Craiova, Romania \\ ${ }^{7}$ Department of Gastroenterology, Faculty of Medicine, University of Medicine and Pharmacy of Craiova, Romania
}

Correspondence should be addressed to Dorin Nicolae Gheorghe; dorinngheorghe@gmail.com and Dora Maria Popescu; popescu131@yahoo.com

Received 8 October 2021; Revised 1 November 2021; Accepted 10 November 2021; Published 19 November 2021

Academic Editor: Gaetano Isola

Copyright (c) 2021 Petra Surlin et al. This is an open access article distributed under the Creative Commons Attribution License, which permits unrestricted use, distribution, and reproduction in any medium, provided the original work is properly cited.

The study is aimed at assessing the impact that periodontal disease and chronic hepatitis $\mathrm{C}$ could have on gingival crevicular fluid levels of the NLRP3 inflammasome, caspase-1 (CASP-1), and interleukin-18 (IL-18) and at evaluating whether the increased local inflammatory reaction with clinical periodontal consequences is correlated to their upregulation. Patients were divided into four groups, according to their periodontal status and previously diagnosed hepatitis C, as follows: (i) CHC group, chronic hepatitis C patients; (ii) P group, periodontal disease patients, systemically healthy; (iii) $\mathrm{CHC}+\mathrm{P}$ group, patients suffering from both conditions; and (iv) $\mathrm{H}$ group, systemically and periodontally healthy controls. Gingival crevicular samples were collected for quantitative analysis of the NLRP3 inflammasome, CASP-1, and IL-18. CHC $+\mathrm{P}$ patients expressed the worse periodontal status and the highest NLRP3, CASP-1, and IL-18 levels, the difference being statistically significant $(p<0.05)$. The P group patients also expressed significantly more elevated NLRP3, CASP-1, and IL18 levels, as compared to nonperiodontal patients ( $\mathrm{CHC}$ and $\mathrm{H}$ groups). Chronic hepatitis $\mathrm{C}$ and periodontal disease could have a significant influence on the upregulation of NLRP3 inflammasome and its components, possibly contributing to an increased local inflammatory reaction and clinical periodontal consequences.

\section{Introduction}

Recent research on the complex molecular mechanisms of the inflammatory reaction has led to the development of the "inflammasome" concept, a multiprotein, oligomer compound, governing inflammation in its early stages $[1,2]$. One of these inflammasomes, the NLRP3 complex, has a crucial role in innate immunity and inflammatory mechanisms [3, 4]. It consists of a Nod-like receptor (NLR) that mediates the activation of protease enzymes (Caspase 1 (CASP-1)) and further regulates the expression of pioneer, key, proinflammatory cytokines, such as interleukin-18 (IL-18) [3].

The periodontal inflammatory process, which defines periodontitis $(\mathrm{P})$, can interact with the chronic inflammatory reaction generated by viral hepatic infection, resulting in systemically important pathogenic implications [5]. The activation of the NLRP3 is caused by bacterial stimuli, as lipopolysaccharides (from Porphyromonas gingivalis) and 
bacterial RNA or by endogenous ones, as extracellular ATP, uric acid, or cholesterol crystals [3]. Despite its elevated concentrations within epithelial tissues, such as the gingival epithelium [6], the NLRP3 inflammasome has received little scientific attention from periodontal researchers, as part of the pathogenic mechanisms governing $\mathrm{P}$ alone, or in association with systemic diseases [7-9]. A recent study by Hernandez et al. highlighted the upregulation of the NLPR3 inflammasome in patients with periodontitis and uncontrolled type 2 diabetes [8]. This could also be the case in patients with periodontitis and cardiovascular diseases, as highlighted by Mahendra et al. [10]. The activation of the NLRP3 inflammasome during periodontal inflammation has been illustrated by using samples of serum and saliva, by Isola et al. [11]. Thus, the study of this particular inflammasome (NLRP3) and its components (CASP-1 and IL-18) was chosen as a focus point of our research, considering the promising results of its assessment as a potential biomarker for the periodontal clinical status [2] and its upregulation in periodontal patients with uncontrolled type 2 diabetes $[6,8,11]$.

Chronic hepatitis $\mathrm{C}(\mathrm{CHC})$ patients can often manifest important oral health issues, that can have a negative impact on their life's quality, adding to the pathological manifestations of the liver disease and its complications [12]. When seeking dental treatments, CHC patients may face various elements of difficulty, such as high personal anxiety or modified healing and recovery processes after dental and periodontal surgery, that limit the complexity of therapeutical options [13]. Corroborated with possible behavioral particularities, CHC patients may comprise more risk factors for the onset of $\mathrm{P}$, leading to its clinical manifestation, triggered by the accumulation of subgingival bacterial plaque deposits [5, 14].

In essence, both $\mathrm{CHC}$ and $\mathrm{P}$ generate a chronic inflammatory reaction, such a pathologic event being driven by proinflammatory mediators that control its extent and intensity $[15,16]$. Our previously published study, focusing on the gingival crevicular fluid (GCF) assessment of interleukin-1alpha's and interleukin-1beta's involvement in the pathogenic process of periodontitis patients with chronic hepatitis $\mathrm{C}$, highlighted a significantly worsened periodontal status and increased levels of these cytokines in patients with both diseases, as compared to those of nonhepatitis C patients suffering from periodontitis [17]. This suggests the negative impact that hepatic pathology may have on local periodontal inflammation.

Hence, the purpose of the present study was to evaluate the GCF levels of the NLRP3 inflammasome and its components in patients with $\mathrm{P}$ and $\mathrm{CHC}$ and to determine if its upregulation is influenced by these joined pathologies, periodontitis, and chronic hepatitis $\mathrm{C}$, leading to a more exacerbated manifestation of periodontal disease in $\mathrm{CHC}$ patients.

\section{Materials and Methods}

2.1. Study Design. The study's design was approved by the Ethical Research Committees at the University of Medicine and Pharmacy of Craiova, Romania, and at the Craiova
Emergency County Hospital fulfilling the requirements of the European Union's General Data Protection Regulation (GDPR) on patient data protection and discretion and the 1975-2013 Declaration of Helsinki. The study approached two main research directions: the first one, clinical and metabolic, consisting of assessment of the patient's periodontal status and certain metabolic parameters, which reflected their systemic and hepatic status; the second direction, immunological, consisting of a quantitative determination of the targeted inflammatory markers within the patients' gingival crevicular samples. The data resulting from the two study directions was subjected to intra- and intergroup statistical analysis for significance and correlation identification.

2.2. Patient Selection. Chronic hepatitis C participants were selected from patients attending the Gastroenterology Clinic of the Craiova Emergency County Hospital, while nonhepatitis $\mathrm{C}$ participants were selected from the patients addressing the Periodontology Department of the Dental Medicine Faculty of the University of Medicine and Pharmacy of Craiova. For inclusion, all hepatitis $\mathrm{C}$ patients had asymptomatic forms of disease. All periodontal patients had to meet the diagnosis criteria issued during the 1996 World Workshop in Clinical Periodontics: (i) minimum of 20 existing natural teeth, (ii) minimum of six periodontal pockets in two different quadrants (probing depth $\geq 5 \mathrm{~mm}$ ), (iii) bleeding on probing, and (iv) minimum one mm clinical attachment loss [18]. According to the 2018 new classification system of periodontal diseases [19], this would correspond to Stage II (moderate) and Stage III (severe) periodontitis, in terms of severity and Grade A (slow) in terms of rate of progression. For inclusion in the healthy control group, patients had to show no symptoms and history of periodontal or gingival disease (no periodontal pockets/bone loss, no gingival bleeding) and no declared systemic diseases.

The exclusion criteria consisted of (i) anti-inflammatory or other type of medication taken in the last 30 days prior to examination, (ii) previous antiviral anti-HCV therapy, (iii) antibiotic treatment taken in the last 90 days prior to examination, (iv) pregnancy, (v) active smoking status, and (vi) other associated systemic diseases.

Upon applying all of the above inclusion/exclusion criteria and obtaining the informed and written individual consent for entering the study, the 62 participating patients were divided into four study groups: (i) $\mathrm{CHC}+\mathrm{P}$ group: 18 patients (aged from 54 to 79 years) suffering from both $\mathrm{CHC}$ and P; (ii) CHC group: 14 patients (aged from 50 to 61 years), suffering only from CHC and being periodontally healthy; (iii) P group: 15 patients (aged from 42 to 63 years), suffering only from $\mathrm{P}$ and being systemically healthy; and (iv) $\mathrm{H}$ group: 15 controls (aged from 40 to 61 years), periodontally and systemically healthy patients.

2.3. Dental and Periodontal Assessment. All participating patients were subjected to a complete oral and periodontal examination, which was used for the diagnosis of possible periodontal conditions. During this examination, the level of dental hygiene was evaluated, by using the O'Leary Index [20]. 
After the assessment of the oral hygiene level, an ultrasonic scaling procedure of calculus and plaque deposits' removal was conducted when required, in order to enable unbiased and undisturbed periodontal probing. The periodontal measurements were performed by using manual University of North Carolina probes (Hu-Friedy, Chicago, Illinois, USA). The periodontal probing provided periodontal parameters such as (i) periodontal probing depth (PD) - in six points for each tooth (messial, central, and distal for the buccal and oral aspects of the teeth), (ii) clinical attachment loss (AL), and (iii) bleeding on probing index (BPI). All periodontal probing measurements were performed by a single, calibrated, examiner. The attachment loss was calculated for each probing site by using the formula: periodontal probing depth $(\mathrm{mms})$ - gingival $\operatorname{margin}(\mathrm{mms})=\operatorname{attachment~level~}(\mathrm{AL}, \mathrm{mms})$.

2.4. Metabolic and Hepatic Assessment. From each patient, a blood sample was collected for the laboratory tests required for the assessment of their metabolic status. This included standard laboratory blood tests such as (i) level of serum glucose-glycemia, as indicator for the glucidic metabolism (reference range 90-110 mg/dL) [21]; (ii) level of total cholesterol, as indicator for the lipidic metabolism (reference range $140-180 \mathrm{mg} / \mathrm{dL}$ ) [21]; and (iii) level of triglycerides, as a complementary indicator for lipidic metabolism (reference range 140-160 mg/dL) [21]. Specific parameters which allowed the assessment of the hepatic function were also tested (i) aspartate aminotransferase (AST) (reference range 6-34 IU/L) [22], (ii) alanine transaminase (ALT) (reference range 20-60IU/L) [22], and (iii) gamma-glutamyl transferase (GGT) (reference range 8-38 IU/L) [22]. The hepatic status was also evaluated through the use of ultrasound elastography imaging test (FibroScan 530, Echosens, Paris, France) for the degree of liver fibrosis in $\mathrm{CHC}$ patients. The scale used to assess the level of liver fibrosis was from zero (= absent) to four (= liver cirrhosis) [23].

2.5. Gingival Crevicular Fluid Sampling. After the supragingival plaque was removed with sterile cotton bullets, gingival crevicular fluid (GCF) was sampled from each of the 62 patients, by using absorbent paper strips (PerioPaper, Oraflow Inc., Smithtown, NY, USA). The two teeth with the deepest pocket depths were selected for sampling, using different paper strips for each tooth, inserted simultaneously at the two teeth. The paper strips were inserted within the periodontal pocket until mild resistance was felt and kept in place for 30 seconds. Upon removal, the strips were visually inspected for blood stains. In order to prevent the strips' contamination with saliva or blood, absorbent cotton roll isolation and air suction were used during the sampling procedure. The quantity of sampled GCF was standardized using the Periotron 8000 device (Oraflow Inc., Smithtown, NY, USA). Afterwards, the paper strips originating from both teeth were pooled together into a plastic microtube containing saline buffer solution (PBS). The sampling procedures were additionally repeated two times. Thus, three microtubes were obtained for each patient, corresponding to the three targeted mediators. The microtubes were preserved at -20 degrees Celsius, until analysis.

2.6. Immunological Assessment. After the GCF samples were collected from all 62 patients, they were transferred to the Immunology Laboratory of the University of Medicine and Pharmacy of Craiova for specific preparation and assessment. For the detection of the targeted inflammatory mediators (NLRP3 inflammasome, CASP-1, and IL-18) within the gingival fluid, the enzyme-linked immunosorbent assay (ELISA) method was used. Specifically designed commercial test kits were used for each of the mediators, (i) NLRP3-OKEH03368 (Aviva Systems Biology, San Diego, USA) (range 0.312-20 ng/mL), (ii) CASP1-OKEH01146 (Aviva Systems Biology, San Diego, USA) (range $15.6-1000 \mathrm{pg} / \mathrm{mL}$ ), and (iii) IL-18-OKCD00106 (Aviva Systems Biology, San Diego, USA) (range: $15.6-1000 \mathrm{pg} / \mathrm{mL}$ ), according to the manufacturer's indications and prescribed work method. The ELISA method was performed with a standard optical analyzer, at $450 \mathrm{~nm}$ wave length.

2.7. Statistical Analysis. All data was centralized and expressed as mean and standard deviation. Afterwards, it was subjected to statistical analysis (GraphPad Software, LLC, San Diego, CA, USA) in order to detect the differences between patients with $\mathrm{CHC}+\mathrm{P}$ and $\mathrm{CHC}, \mathrm{CHC}+\mathrm{P}$ and $\mathrm{P}$, and $\mathrm{CHC}$ and $\mathrm{P}$ groups, using Mann-Whitney $U$ test (statistically significant at 5\%, two-tailed). For categorical variables, the comparisons between the groups were evaluated using the Fisher's exact test. The existence of statistical correlations between the different types of data was assessed using Pearson coefficients. A power computation ( $\mathrm{G} *$ Power 3, University of Dusseldorf, Germany) was completed and revealed that, for our samples, the effect size was large (1.468) and power equal to 0.979 (alpha equal to 0.05 ).

\section{Results}

3.1. Demographics, Glucose, and Liver Fibrosis Level. There was no statistical difference regarding the average age of the study's participants or the gender distribution among the study groups $(p>0.05)$ (Table 1$)$. There was no significant difference concerning the level of serum glucose among the test groups. In the hepatitis $\mathrm{C}$ patients (groups $\mathrm{CHC}+\mathrm{P}$ and $\mathrm{CHC}$ ), there was a similar level of liver fibrosis (predominatly $\mathrm{F} 1,61.11 \%$ in the $\mathrm{CHC}+\mathrm{P}$ group and $64.28 \%$ in the $\mathrm{CHC}$ group), with no significant difference $(p>0.05)$. The $\mathrm{CHC}+\mathrm{P}$ group included significantly more patients with F2 stage liver fibrosis than the CHC group (Table 1).

3.2. Patients with $\mathrm{CHC}+P$ Expressed the Most Severely Modified Clinical Periodontal Status. The patients of the $\mathrm{CHC}+\mathrm{P}$ group showed a significantly worsened periodontal status as compared to $\mathrm{P}$ patients, in terms of periodontal probing depth and clinical attachment loss (Table 2). A statistically significant difference was also found between the plaque index and gingival bleeding index of the $\mathrm{CHC}$ $+\mathrm{P}$ and $\mathrm{P}$ groups (Table 2). 
TABle 1: Study's demographic characteristics, serum glucose level, liver fibrosis, and statistical significance ( $p$ value) for differences between test groups.

\begin{tabular}{|c|c|c|c|c|c|c|c|}
\hline \multirow{2}{*}{ Parameter } & \multicolumn{4}{|c|}{ Groups } & \multirow{2}{*}{$p \mathrm{CHC}+\mathrm{P}$ vs. $\mathrm{CHC}$} & \multirow{2}{*}{$p \mathrm{CHC}+\mathrm{P}$ vs. $\mathrm{P}$} & \multirow{2}{*}{$p$ CHC vs. $\mathrm{P}$} \\
\hline & $\mathrm{CHC}+\mathrm{P}$ & $\mathrm{CHC}$ & $\mathrm{P}$ & $\mathrm{H}$ & & & \\
\hline Age (years) & 62.61 & 57.76 & 55.86 & 50.13 & $>0.05$ & $>0.05$ & $>0.05$ \\
\hline Gender (male/female) & $8 / 10$ & $5 / 9$ & $8 / 7$ & $6 / 9$ & $>0.05$ & $>0.05$ & $>0.05$ \\
\hline Glucose $(\mathrm{mg} / \mathrm{dl}) \pm \mathrm{SD}$ & $108.83 \pm 27.22$ & $103.14 \pm 16.54$ & $98.2 \pm 13.43$ & $95.53 \pm 8.75$ & $>0.05$ & $>0.05$ & $>0.05$ \\
\hline Liver fibrosis (\%) & & & - & - & & - & - \\
\hline $\mathrm{F} 1$ & 61.11 & 64.28 & & & $>0.05$ & & \\
\hline F2 & 22.22 & 7.14 & & & $<0.05$ & & \\
\hline F3 & 11.11 & 14.28 & & & $>0.05$ & & \\
\hline $\mathrm{F} 4$ & 5.55 & 14.28 & & & $>0.05$ & & \\
\hline
\end{tabular}

TABLe 2: Study's clinical results and statistical significance ( $p$ value) for differences between test groups.

\begin{tabular}{|c|c|c|c|c|c|c|c|}
\hline \multirow{2}{*}{ Parameter } & \multicolumn{4}{|c|}{ Groups } & \multirow{2}{*}{$\begin{array}{l}p \mathrm{CHC}+\mathrm{P} \\
\text { vs. } \mathrm{CHC} \\
\end{array}$} & \multirow{2}{*}{$\begin{array}{c}p \mathrm{CHC}+\mathrm{P} \\
\text { vs. } \mathrm{P}\end{array}$} & \multirow{2}{*}{$\begin{array}{c}p \mathrm{CHC} \\
\text { vs. } \mathrm{P}\end{array}$} \\
\hline & $\mathrm{CHC}+\mathrm{P}$ & $\mathrm{CHC}$ & $\mathrm{P}$ & $\mathrm{H}$ & & & \\
\hline Periodontal probing depth $(\mathrm{mm}) \pm \mathrm{SD}$ & $3.85 \pm 0.92$ & $1.45 \pm 0.2$ & $3.76 \pm 0.53$ & $1.24 \pm 0.2$ & $<0.05$ & $<0.05$ & $<0.05$ \\
\hline $\begin{array}{l}\text { Clinical attachment } \\
\text { loss }(\mathrm{mm}) \pm \mathrm{SD}\end{array}$ & $5.42 \pm 1.61$ & $1.83 \pm 0.42$ & $3.98 \pm 0.51$ & $0.52 \pm 0.19$ & $<0.05$ & $<0.05$ & $<0.05$ \\
\hline Plaque index $(\%) \pm S D$ & $57.5 \pm 17.27$ & $25.85 \pm 13.63$ & $39.33 \pm 12.61$ & $13.86 \pm 4.03$ & $<0.05$ & $<0.05$ & $<0.05$ \\
\hline $\begin{array}{l}\text { Gingival bleeding } \\
\text { index }(\%) \pm S D\end{array}$ & $50.53 \pm 11.98$ & $7.92 \pm 5.31$ & $43.6 \pm 13.18$ & $7.4 \pm 2.35$ & $<0.05$ & $<0.05$ & $<0.05$ \\
\hline
\end{tabular}

3.3. Patients with $\mathrm{CHC}+\mathrm{P}$ Exhibited Significantly Elevated Levels of Proinflammatory Mediators. The highest values of the assessed mediators were recorded for the $\mathrm{CHC}+\mathrm{P}$ group, being significantly more elevated that those of the other groups (Table 3 ). Both groups of periodontitis patients $(\mathrm{CHC}+\mathrm{P}$ and $\mathrm{P})$ expressed significantly elevated levels for all of the three assessed proinflammatory mediators (NLRP3, CASP1, and IL-18) as compared to the nonperiodontitis groups ( $\mathrm{CHC}$ and $\mathrm{H}$ ) (Table 3 ). The proinflammatory mediators' levels in all three test groups were significantly more elevated than in controls (Table 3 ).

\subsection{NLRP3, CASP1, and IL-18 GCF Levels Associated Positively with Certain Periodontal and Metabolic} Parameters. The results delivered significant positive correlations between the GCF NLRP3 levels and the periodontal probing depth, clinical attachment loss, and gingival bleeding index $(r=0.4 ; p<0.05)$ (Table 4). The NLRP3 GCF levels also positively correlated with certain metabolic parameters, including the glucose, aspartatetransferase (AST), and alaninetransferase (ALT) levels. Other significant positive correlations were found between the CASP1 GCF levels and the age of the patients, the clinical attachment loss, gingival bleeding index, and ALT level (Table 4). The GCF IL-18 levels significantly correlated with the age of patients, the periodontal probing depth, the clinical attachment loss, the gingival bleeding index, and triglyceride level (Table 4). No correlations were identified between the level of liver fibrosis and the assessed proinflammatory mediators.

\section{Discussion}

The development of the "inflammasome" concept has opened new perspectives for periodontal inflammation research. NLPR3 is a key element of this inflammatory reaction, being considered as its pioneer triggering factor, from the first initial contact with bacterial antigens, such as Porphyromonas gingivalis' lipopolysaccharides (LPS) [24-26]. While some authors state that LPS can stimulate NLRP3 synthesis by activation of the Toll-like 4 receptor [27], others have observed that the inflammasome decreases its activity when in contact with subgingival bacteria [28]. In our study, there was no correlation between the GCF NLRP3 levels and the level of bacterial plaque. However, certain studies reported that NLRP3 expression is more elevated in periodontitis patients than in healthy controls [29], both in specific cells [8], and within saliva [11,30]. Our study delivered similar results, highlighting the significant differences of the GCF NLRP3 levels between the $\mathrm{P}$ and $\mathrm{H}$ groups and between the $\mathrm{CHC}+\mathrm{P}$ and $\mathrm{CHC}$ ones, which suggest that periodontal pathological events trigger a considerable increase of NLRP3 expression. This hypothesis was also assessed and endorsed by a recent review on the subject, which concluded that periodontal diseases can be characterized by an upregulation of inflammasomes, alongside with a downregulation of their inhibitor proteins [29].

The statistical results of our study highlighted significant correlations between the GCF NLRP3 levels and serum glucose levels of the participating patients, in accordance with 
TABLE 3: Study's immunological results and statistical significance ( $p$ value) for differences between test groups.

\begin{tabular}{|c|c|c|c|c|c|c|c|}
\hline \multirow{2}{*}{ Parameter } & \multicolumn{4}{|c|}{ Groups } & \multirow{2}{*}{$p \mathrm{CHC}+\mathrm{P}$ vs. $\mathrm{CHC}$} & \multirow{2}{*}{$p \mathrm{CHC}+\mathrm{P}$ vs. $\mathrm{P}$} & \multirow[b]{2}{*}{$p$ CHC vs. $\mathrm{P}$} \\
\hline & $\mathrm{CHC}+\mathrm{P}$ & $\mathrm{CHC}$ & $\mathrm{P}$ & $\mathrm{H}$ & & & \\
\hline $\mathrm{NLRP} 3(\mathrm{ng} / \mathrm{mL}) \pm \mathrm{SD}$ & $1.534 \pm 0.32$ & $0.989 \pm 0.3$ & $1.251 \pm 0.36$ & $0.583 \pm 0.18$ & $<0.05$ & $<0.05$ & $<0.05$ \\
\hline $\mathrm{CASP} 1(\mathrm{ng} / \mathrm{mL}) \pm \mathrm{SD}$ & $0.369 \pm 0.14$ & $0.2 \pm 0.07$ & $0.283 \pm 0.08$ & $0.05 \pm 0.04$ & $<0.05$ & $<0.05$ & $<0.05$ \\
\hline $\mathrm{IL}-18(\mathrm{ng} / \mathrm{mL}) \pm \mathrm{SD}$ & $0.287 \pm 0.07$ & $0.193 \pm 0.05$ & $0.2 \pm 0.03$ & $0.075 \pm 0.07$ & $<0.05$ & $<0.05$ & $<0.05$ \\
\hline
\end{tabular}

TABLE 4: Synopsis of correlation statistical assessment (Pearson's test) for whole patient batch between NLRP3, CASP-1, and IL-18 levels and assessed parameters.

\begin{tabular}{lccc}
\hline \multirow{2}{*}{ Parameter } & \multicolumn{3}{c}{ Proinflammatory } \\
& NRLP3 & CASP1 & IL-18 \\
& $r / p$ & $r / p$ & $r / p$ \\
\hline Gender & $0.05 / 0.8$ & $0.08 / 0.71$ & $0.14 / 0.52$ \\
Age & $0.33 / 0.12$ & $0.56 / 0.005^{*}$ & $0.43 / 0.04^{*}$ \\
Periodontal probing depth & $0.45 / 0.03^{*}$ & $0.39 / 0.06$ & $0.47 / 0.02^{*}$ \\
Clinical attachment loss & $0.52 / 0.01^{*}$ & $0.46 / 0.02^{*}$ & $0.52 / 0.013^{*}$ \\
Plaque index & $0.27 / 0.21$ & $0.36 / 0.09$ & $0.4 / 0.06$ \\
Gingival bleeding index & $0.45 / 0.03^{*}$ & $0.48 / 0.02^{*}$ & $0.42 / 0.04^{*}$ \\
Glucose & $0.43 / 0.04^{*}$ & $0.14 / 0.51$ & $0.03 / 0.86$ \\
Cholesterol & $0.02 / 0.9$ & $0.26 / 0.22$ & $0.39 / 0.06$ \\
Triglycerides & $0.23 / 0.28$ & $0.05 / 0.79$ & $0.42 / 0.04^{*}$ \\
Aspartate transaminase & $0.45 / 0.04^{*}$ & $0.14 / 0.52$ & $0.14 / 0.5$ \\
Alanine transaminase & $0.51 / 0.02^{*}$ & $0.57 / 0.02^{*}$ & $0.08 / 0.69$ \\
Glutamyl transferase & $0.2 / 0.35$ & $0.18 / 0.4$ & $0.05 / 081$ \\
\hline
\end{tabular}

$r$ : Pearson's $r$; $p$ : statistical significance. ${ }^{*}$ Statistically significant value $(p<0.05)$.

other studies on the topic of NLRP3 inflammasome, periodontal disease, and diabetes association $[9,11,31-34]$. This correlation endorses the pathogenic links existing between periodontal diseases and cellular resistance to insulin [35]. Additional pathogenic connections also lay between chronic hepatitis C and insulin resistance [36]. These elements suggest that this pathologic mechanism could bridge the connection between chronic hepatitis and periodontal disease, through the significant impact and bi-directional consequences it inflicts on the inflammatory reaction.

The immunological analysis performed in our study generated comparable outputs on the average values of CASP1 within the GCF samples of periodontitis patients, which were significantly higher than those of healthy controls [37]. Histologically, CASP1 is predominantly expressed within gingival epithelial cells, as keratinocytes, and connective tissue cells, being almost absent when the periodontal tissues are not inflamed [37-39]. Moreover, important periodontal bacterial pathogens (Aggregatibacter actinomycetemcomitans and Porphyromonas gingivalis) have been shown to trigger caspase expression within epithelial cells and macrophages [40,41]. Within this finding, in our study also, a significant positive correlation was identified between the GCF CASP1 levels and the clinical periodontal parame- ters used for assessing the severity of periodontal damage (clinical attachment loss and gingival bleeding index).

Our current study's results show, as similar other ones, that the GCF IL-18 levels were significantly higher in the samples of periodontal patients as compared to healthy controls [42-45]. Moreover, the statistical analysis denoted significant correlations between the GCF IL-18 levels and the parameters of periodontal disease's severity (periodontal probing depth, clinical attachment loos, and gingival bleeding index), similar to those highlighted by other papers [29]. IL-18 has also been suggested as a possible indicator for periodontal structures' damage [45]. In accordance with this finding, our $\mathrm{CHC}+\mathrm{P}$ patients expressed significantly elevated GCF IL-18, reflecting their unfavorable clinical periodontal status.

Regarding the hepatic pathology, NLRP3 has been observed to activate when stimulated by the hepatitis $\mathrm{C}$ virus, within white and red blood cells [46-48]. Moreover, in an in vitro setting, the presence of the virus determined cellular pyroptosis within infected hepatocytes, an event mainly controlled by NLRP3 and CASP1 activity [47]. NLRP3 is also involved in the onset of the chronic hepatic inflammatory reaction, consequent to $\mathrm{HCV}$ infection, together with IL-1 $\beta$ [48]. Thus, CHC patients are expected to exhibit elevated serum NLRP3 levels. This fact has also been shown by the results of our study that identified more elevated GCF NLRP3 levels in the samples of CHC patients, that those of the $\mathrm{H}$ group, given that GCF is a blood serum derivate. As shown by the delivered results, when $\mathrm{CHC}$ patients also suffer from P, their GCF NLRP3 levels increased significantly compared to those of patients suffering from $\mathrm{CHC}$ or $\mathrm{P}$ alone, suggesting that NLRP3 expression is upregulated when the two diseases occur in the same patient.

CASP1 is also involved in the pathological processes of $\mathrm{CHC}$, as the HCV-infected cells are able to synthesis and release the NLRP3 inflammasome [49, 50]. Our study's results highlighted more elevated average GCF CASP1 levels in the samples of $\mathrm{CHC}+\mathrm{P}$ patients, as compared to $\mathrm{P}$ patients. Significant differences were also identified between the average values of the $\mathrm{CHC}$ patients, with and without periodontal disease. These results suggest the significant impact that chronic inflammation, either hepatic or periodontal, could have on the GCF CASP1 levels.

Concerning the hepatic disease, IL-18 also has immunological and clinical implications on $\mathrm{CHC}$, as affected patients often exhibit significantly elevated values of this mediator, compared to healthy controls [51-54]. In our study, significant differences of the GCF IL-18 levels were recorded 
between the $\mathrm{CHC}+\mathrm{P}$ group and the $\mathrm{P}$ group, endorsing the negative impact that HCV infection can have on the periodontal status, in similar periodontal pathological settings. This can amplify gingival pathogenic events and trigger a more intense and severe periodontal inflammatory reaction.

Our results show that $\mathrm{CHC}+\mathrm{P}$ patients exhibited a more severely modified clinical periodontal status. In addition, these patients also exhibited the highest NLRP3 GCF levels. The negative character of their periodontal status could be in relation to the upregulation of this mediator. A study by Garcia-Hernandez et al. stated that the upregulated NLRP3 levels could increase the inflammatory response in uncontrolled type-2 diabetes periodontal patients [8]. This was also shown by a recent study by Isola et al., in samples of saliva and serum, originating from patients with periodontitis and diabetes [11]. Concerning $\mathrm{CHC}$ periodontal patients, future extended research is required to test this hypothesis.

Given the sample size limitations, all three assessed elements (NLRP3, CASP1, and IL-18) expressed significantly elevated GCF levels in $\mathrm{CHC}+\mathrm{P}$ patients, as compared to the other groups, suggesting that the coexisting hepatic pathology might have an upregulating effect on these proinflammatory mediators. The decline of the clinical periodontal and immunological status of these patients should be taken into account when developing holistic therapeutical strategies.

\section{Conclusions}

Within the limitations of this study, we could conclude that chronic hepatitis $\mathrm{C}$ and periodontitis might have a joined effect on the upregulation of the NLRP3 inflammasome and its components in the GCF. This fact could justify the exacerbated manifestation of periodontal disease in chronic hepatitis C patients. This motivates future expanded research on the matter.

\section{Data Availability}

The data used to support the findings of this study are available from the corresponding author upon reasonable request.

\section{Conflicts of Interest}

The authors declare no conflict of interest.

\section{Authors' Contributions}

Luminita Lazar, Cerasella Sincar, Mihail Boldeanu, Allma Pitru, and Cristina Florescu share equal contribution to that of the first author, thus can be considered as main authors.

\section{References}

[1] J. T. Marchesan, M. S. Girnary, K. Moss et al., "Role of inflammasomes in the pathogenesis of periodontal disease and therapeutics," Periodontology 2000, vol. 82, no. 82, pp. 93-114, 2000.
[2] D. M. Isaza-Guzmán, V. M. Medina-Piedrahíta, C. GutiérrezHenao, and S. I. Tobón-Arroyave, "Salivary levels of NLRP3 inflammasome-related proteins as potential biomarkers of periodontal clinical status," Journal of Periodontology, vol. 88, no. 12, pp. 1329-1338, 2017.

[3] N. Kelley, D. Jeltema, Y. Duan, and Y. He, "The NLRP3 inflammasome: an overview of mechanisms of activation and regulation," International Journal of Molecular Sciences, vol. 20, no. 13, p. 3328, 2019.

[4] L. Wang and A. V. Hauenstein, "The NLRP3 inflammasome: mechanism of action, role in disease and therapies," Molecular Aspects of Medicine, vol. 76, article 100889, 2020.

[5] P. Surlin, D. N. Gheorghe, L. Foia, A. Surdu, and I. Rogoveanu, "Periodontal Implications of Hepatitis C Infection," in Hepatitis C: From Infection to Cure, I. Shalid, Ed., pp. 119-138, IntechOpen, Zagreb, Croatia, 2018.

[6] P. Zhang, B. Lu, R. Zhu et al., "Hyperglycemia accelerates inflammaging in the gingival epithelium through inflammasomes activation," J. Periodcaontal Res., vol. 56, no. 4, pp. 667-678, 2021.

[7] F. Xue, R. Shu, and Y. Xie, "The expression of NLRP3, NLRP1 and AIM2 in the gingival tissue of periodontitis patients: RTPCR study and immunohistochemistry," Archives of Oral Biology, vol. 60, no. 6, pp. 948-958, 2015.

[8] A. L. García-Hernández, A. E. Muñoz-Saavedra, P. GonzálezAlva et al., "Upregulation of proteins of the NLRP3 inflammasome in patients with periodontitis and uncontrolled type 2 diabetes," Oral Diseases, vol. 25, no. 2, pp. 596-608, 2019.

[9] K. Aral, E. Berdeli, P. R. Cooper et al., "Differential expression of inflammasome regulatory transcripts in periodontal disease," Journal of Periodontology, vol. 91, no. 5, pp. 606-616, 2020.

[10] J. Mahendra, A. N. Rao, L. Mahendra et al., "Genetic polymorphisms of NLRP3 (rs4612666) and CARD8 (rs2043211) in periodontitis and cardiovascular diseases," Biology, vol. 10, no. 7, p. 592, 2021.

[11] G. Isola, A. Polizzi, S. Santonocito, A. Alibrandi, and R. C. Williams, "Periodontitis activates the NLRP3 inflammasome in serum and saliva," Journal of Periodontology, 2021.

[12] E. A. Coates, D. Brennan, R. M. Logan et al., "Hepatitis C infection and associated oral health problems," Australian Dental Journal, vol. 45, no. 2, pp. 108-114, 2000.

[13] V. Brailo, I. Pelivan, J. Škaričić, M. Vuletić, N. Dulčić, and G. Cerjan-Letica, "Treating patients with HIV and hepatitis $\mathrm{B}$ and $\mathrm{C}$ infections: Croatian dental students' knowledge, attitudes, and risk perceptions," Journal of Dental Education, vol. 75, pp. 1115-1126, 2011.

[14] C. Treloar, J. Rance, and M. Backmund, "Understanding barriers to hepatitis $\mathrm{C}$ virus care and stigmatization from a social perspective," Clinical Infectious Diseases, vol. 57, supplement 2, pp. S51-S55, 2013.

[15] T. D. F. Borges, S. C. Regalo, M. Taba Jr., S. Siéssere, W. Mestriner Jr., and M. Semprini, "Changes in masticatory performance and quality of life in individuals with chronic periodontitis," Journal of Periodontology, vol. 84, no. 3, pp. 325-331, 2013.

[16] B. W. Chaffee and S. J. Weston, “Association between chronic periodontal disease and obesity: a systematic review and metaanalysis," Journal of Periodontology, vol. 81, no. 12, pp. 1708$1724,2010$.

[17] P. Surlin, D. N. Gheorghe, D. M. Popescu et al., "Interleukin- $1 \alpha$ and $-1 \beta$ assessment in the gingival crevicular fluid 
of periodontal patients with chronic hepatitis C," Experimental and Therapeutic Medicine, vol. 20, no. 3, pp. 23812386, 2020.

[18] G. C. Armitage, "Development of a classification system for periodontal diseases and conditions," Annals of Periodontology, vol. 4, no. 1, pp. 1-6, 1999.

[19] M. S. Tonetti, H. Greenwell, and K. S. Kornman, "Staging and grading of periodontitis: framework and proposal of a new classification and case definition," Journal of Periodontology, vol. 89, Supplement 1, pp. S159-S172, 2018.

[20] T. J. O'Leary, R. B. Drake, and J. E. Naylor, "The plaque control record," Journal of Periodontology, vol. 43, no. 1, pp. 38-38, 1972.

[21] A. C. Guyton and J. E. Hall, "Metabolism and Temperature Regulation," in Textbook of Medical Physiology, A. C. Guyton and J. E. Hall, Eds., pp. 829-901, Sauders/Elsevier, Philadelphia, 11th ed. edition, 2006.

[22] D. S. Pratt, "Liver Chemistry and Function Tests," in Sleisenger and Fordtran's Gastrointestinal and Liver Disease: Pathophysiology, Diagnosis, Management, M. Feldman, L. S. Friedman, and M. H. Sleisenger, Eds., pp. 1243-1254, Saunders/Elsevier, Philadelphia, 10th edition, 2016.

[23] P. Bedossa and T. Poynard, "An algorithm for the grading of activity in chronic hepatitis C," Hepatology, vol. 24, no. 2, pp. 289-293, 1996.

[24] G. Hajishengallis, S. Liang, M. A. Payne et al., "Low-abundance biofilm species orchestrates inflammatory periodontal disease through the commensal microbiota and complement," Cell Host \& Microbe, vol. 10, no. 5, pp. 497-506, 2011.

[25] S. Kim, M. H. Park, Y. R. Song, H. S. Na, and J. Chung, "Aggregatibacteractinomycetemcomitans-induced AIM2 inflammasome activation is suppressed by xylitol in differentiated THP-1 macrophages," Journal of Periodontology, vol. 87, no. 6, pp. e116-e126, 2016.

[26] E. Park, H. S. Na, Y. R. Song, S. Y. Shin, Y. M. Kim, and J. Chung, "Activation of NLRP3 and AIM2 Inflammasomes by Porphyromonas gingivalis infection," Infection and Immunity, vol. 82, no. 1, pp. 112-123, 2014.

[27] G. N. Belibasakis, B. Guggenheim, and N. Bostanci, "Downregulation of NLRP3 inflammasome in gingival fibroblasts by subgingival biofilms: involvement of Porphyromonas gingivalis," Journal of Innate Immunity, vol. 19, no. 1, pp. 3-9, 2013.

[28] Ö. Yilmaz, A. A. Sater, L. Yao, T. Koutouzis, M. Pettengill, and D. M. Ojcius, "ATP-dependent activation of an inflammasome in primary gingival epithelial cells infected by Porphyromonas gingivalis," Cellular Microbiology, vol. 12, no. 2, pp. 188-198, 2010.

[29] K. Aral, M. R. Milward, Y. Kapila, A. Berdeli, and P. R. Cooper, "Inflammasomes and their regulation in periodontal disease: a review," Journal of Periodontal Research, vol. 55, no. 4, pp. 473-487, 2020.

[30] D. M. Isaza-Guzmán, M. Hernández-Viana, D. M. BonillaLeón, M. C. Hurtado-Cadavid, and S. I. Tobón-Arroyave, "Determination of _NLRP3___rs4612666_) and _IL-1B_ (_rs1143634_) genetic polymorphisms in periodontally diseased and healthy subjects," Archives of Oral Biology, vol. 65, pp. 44-51, 2016.

[31] B. Vandanmagsar, Y. H. Youm, A. Ravussin et al., "The NLRP3 inflammasome instigates obesity-induced inflammation and insulin resistance," Nature Medicine, vol. 17, no. 2, pp. 179-188, 2011.
[32] D. Jiang, S. Chen, R. Sun, X. Zhang, and D. Wang, "The NLRP3 inflammasome: role in metabolic disorders and regulation by metabolic pathways," Cancer Letters, vol. 419, pp. 8-19, 2018.

[33] A. J. Wolf, C. N. Reyes, W. Liang et al., "Hexokinase is an innate immune receptor for the detection of bacterial peptidoglycan," Cell, vol. 166, no. 3, pp. 624-636, 2016.

[34] X. Huang, X. Yang, J. Ni et al., "Hyperglucose contributes to periodontitis: involvement of the NLRP3 pathway by engaging the innate immunity of oral gingival epithelium," Journal of Periodontology, vol. 86, no. 2, pp. 327-335, 2015.

[35] F. Nishimura, Y. Soga, Y. Iwamoto, C. Kudo, and Y. Murayama, "Periodontal disease as part of the insulin resistance syndrome in diabetic patients," International Academy of Periodontology, vol. 7, pp. 16-20, 2005.

[36] J. M. Hui, A. Sud, G. C. Farrell et al., "Insulin resistance is associated with chronic hepatitis $\mathrm{C}$ and virus infection fibrosis progression," Gastroenterology, vol. 125, no. 6, pp. 1695-1704, 2003.

[37] A. R. Pradeep, D. K. Suke, M. R. Prasad et al., "Expression of key executioner of apoptosis caspase-3 in periodontal health and disease," Journal of Investigative and Clinical Dentistry, vol. 7, no. 2, pp. 174-179, 2016.

[38] F. Q. Bui, L. Johnson, J. Roberts et al., "Fusobacterium nucleatum infection of gingival epithelial cells leads to NLRP3 inflammasome-dependent secretion of IL-1 $\beta$ and the danger signals ASC and HMGB1," Cellular Microbiology, vol. 18, no. 7, pp. 970-981, 2016.

[39] B. Song, T. Zhou, W. L. Yang, J. Liu, and L. Q. Shao, "Programmed cell death in periodontitis: recent advances and future perspectives," Oral Diseases, vol. 23, no. 5, pp. 609619, 2017.

[40] R. Cheng, W. Liu, R. Zhang, Y. Feng, N. A. Bhowmick, and $\mathrm{T}$. Hu, "Porphyromonas gingivalis-Derived lipopolysaccharide combines hypoxia to induce caspase-1 activation in periodontitis," Frontiers in Cellular and Infection Microbiology, vol. 7, p. 474, 2017.

[41] D. Zhao, Y. Wu, J. Zhuang, C. Xu, and F. Zhang, "Activation of NLRP1 and NLRP3 inflammasomes contributed to cyclic stretch-induced pyroptosis and release of IL- $1 \beta$ in human periodontal ligament cells," Oncotarget, vol. 7, no. 42, pp. 68292-68302, 2016.

[42] J. A. Gracie, S. E. Robertson, and I. B. McInnes, "Interleukin18," Journal of Leukocyte Biology, vol. 73, no. 2, pp. 213-224, 2003.

[43] A. R. Pradeep, P. Hadge, S. Chowdhry, S. Patel, and D. Happy, "Exploring the role of Th1 cytokines: interleukin-17 and interleukin-18 in periodontal health and disease," Journal of Oral Science, vol. 51, no. 2, pp. 261-266, 2009.

[44] C. M. Figueredo, B. Rescala, R. P. Teles et al., "Increased interleukin-18 in gingival crevicular fluid from periodontitis patients," Molecular oral microbiology, vol. 23, no. 2, pp. 173-176, 2008.

[45] Ö. Özçaka, A. Nalbantsoy, and N. Buduneli, "Interleukin-17 and interleukin-18 levels in saliva and plasma of patients with chronic periodontitis," Journal of Periodontal Research, vol. 46, no. 5, pp. 592-598, 2011.

[46] W. Chen, Y. Xu, H. Li et al., "HCV genomic RNA activates the NLRP3 inflammasome in human myeloid cells," PloS One, vol. 9, no. 1, article e84953, 2014.

[47] H. M. Kofahi, N. G. A. Taylor, K. Hirasawa, M. D. Grant, and R. S. Russell, "Hepatitis C virus infection of cultured human 
hepatoma cells causes apoptosis and pyroptosis in both infected and bystander cells," Scientific Reports, vol. 6, no. 1, p. 37433, 2016.

[48] A. A. Negash, H. J. Ramos, N. Crochet et al., "IL-1 $\beta$ production through the NLRP3 inflammasome by hepatic macrophages links hepatitis $\mathrm{C}$ virus infection with liver inflammation and disease," PLoS pathogens, vol. 9, article e1003330, 2013.

[49] J. A. Del Campo, P. Gallego, and L. Grande, "Role of inflammatory response in liver diseases: therapeutic strategies," World Journal of Hepatology, vol. 10, no. 1, pp. 1-7, 2018.

[50] D. Burdette, A. Haskett, L. Presser, S. McRae, J. Iqbal, and G. Waris, "Hepatitis C virus activates interleukin- $1 \beta$ via caspase-1-inflammasome complex," The Journal of General Virology, vol. 93, no. 2, pp. 235-246, 2012.

[51] Z. Niu, P. Zhang, and Y. Tong, "Association of plasma interleukin-18 levels and polymorphisms in interleukin-18 gene with outcomes of hepatitis $\mathrm{C}$ virus infections: a metaanalysis," Journal of immunoassay, vol. 36, no. 3, pp. 221232, 2015.

[52] S. Shrivastava, A. Mukherjee, R. Ray, and R. B. Ray, "Hepatitis $\mathrm{C}$ virus induces Interleukin-1(IL-1)/IL-18 in circulatory and resident liver macrophages," Journal of Virology, vol. 87, no. 22, pp. 12284-12290, 2013.

[53] M. A. Chattergoon, J. S. Levine, R. Latanich, W. O. Osburn, D. L. Thomas, and A. L. Cox, "High plasma interleukin-18 levels mark the acute phase of hepatitis C virus infection," The Journal of Infectious Diseases, vol. 204, no. 11, pp. 17301740, 2011.

[54] A. Sharma, A. Chakraborti, A. Das, R. K. Dhiman, and Y. Chawla, "Elevation of interleukin-18 in chronic hepatitis C: implications for hepatitis $\mathrm{C}$ virus pathogenesis," Immunology, vol. 128, 1 part 2, pp. e514-e522, 2009. 